\title{
Effects of Carbon Nanotubes on Processing Stability of Polyoxymethylene in Melt-mixing Process
}

You Zeng, Zhe Ying, Jinhong Du, and Hui-Ming Cheng *

\section{Supporting Information:}

Figure S1 shows the microstructures of the pristine, chemically purified and graphitized CNTs characterized by XRD and Raman spectrum. The graphitized CNTs exhibit the integrated structures attributed to the rearrangement of disordered graphite layers during the annealing treatment at $2600{ }^{\circ} \mathrm{C}$. Comparing with the pristine CNTs, the purified CNTs show the similar amorphous structures, which maybe are associated with the corrosion of CNT-walls by strong acid during the chemical purification, and hence there is no obvious difference in their XRD patterns. Moreover, the representative changes for different CNTs in Raman spectrum are shown in Figure S1b. There are two characteristic bands, one is the $G$ band at ca. $1570 \mathrm{~cm}^{-1}$ and the other is $D$ band at ca. $1330 \mathrm{~cm}^{-1}$; those two bands are attributed to the contributions of the ordered graphite sheets and of the amorphous carbon, respectively; $R$ value, the ratio of intensity of $D$ band to that of $G$ band, can represent the structural irregularity of samples. For the graphitized CNTs, their $R$ value is only 0.29 and is much lower than that of other CNTs; it means the graphitized CNTs have the less defects and more integrated microstructures than the pristine and chemically purified CNTs.
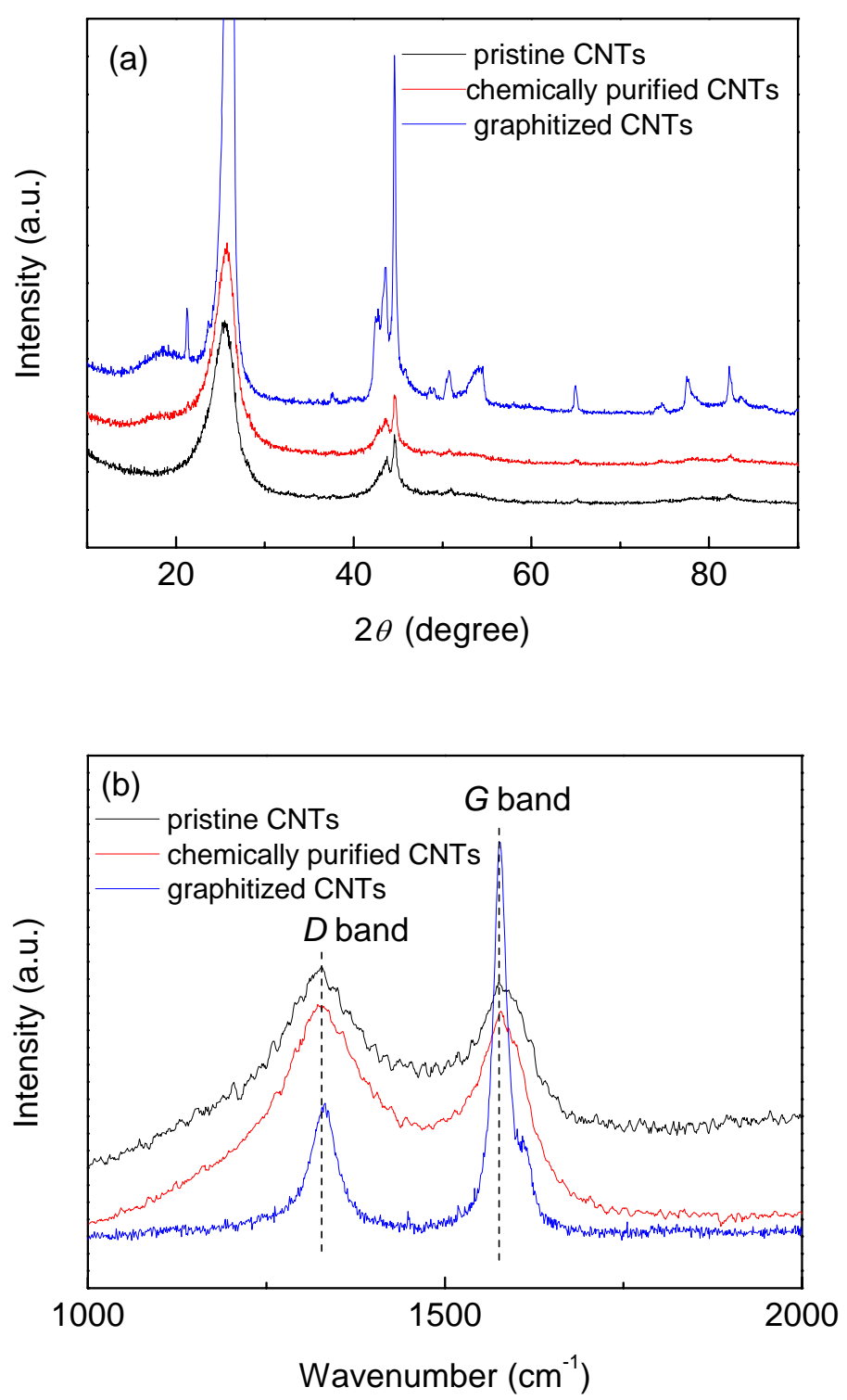

Figure S1. XRD (a) and Raman (b) patterns of the pristine (black), chemically purified (red) and graphitized (blue) CNTs. 